\section{Magma speed dating: syn-eruptive magma mingling determined through major element and volatile exchange of silicate glass}

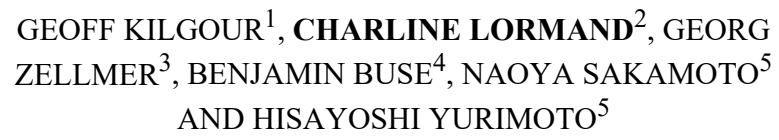

${ }^{1}$ GNS Science

${ }^{2}$ Volcanic Risk Solutions, Massey University

${ }^{3}$ Massey University

${ }^{4}$ University of Bristol

${ }^{5}$ Hokudai University

Presenting Author: c.lormand@massey.ac.nz

Magma mixing plays a key role in triggering many volcanic eruptions. It is often cryptic to the naked eye but revealed by the chemistry of the crystal cargo. Incomplete magma mixing, i.e. mingling, however, is also frequently observed. If injection of new magma into a magma reservoir takes place at shallow depth, diffusion chronometry may be used to tie magmatic mingling timescales to the onset of eruption. The early phase of the 19762000 eruption episode of Whakaari/White Island, New Zealand, was characterized by frequent Strombolian to phreatomagmatic eruptions, and erupted scoria from the 1977 eruption containing evidence of mingling between dacitic and mafic melts. Regions of mingling were targeted using a scanning electron microscope and a secondary ion mass spectrometer equipped with a SCAPS (Stacked CMOS-type Active Pixel Sensor), providing highresolution images of the sharp chemical gradients between the mingled melts. Discrimination of melt compositions was performed using a field emission gun built on an electron probe micro-analyser. Eight profiles across sharp boundaries were extracted for diffusion modelling of two halogens with wellconstrained diffusivities (i.e. chlorine and fluorine). The few microlites found in both melts show morphological differences: the most silica-rich melt contains euhedral crystals, and the silica-poor melt is characterised by acicular (e.g. needle-like) crystals. Large variations in the distribution of volatile elements in the melts are observed at the micrometer scale suggesting extremely short diffusion timescales. Simple and complex (e.g. uphill and downhill volatile diffusion) mingled textures are widespread across the scoria, and thus highlight the chaotic behavior of magma mingling. The dry and high temperature nature of Whakaari magmas results in rapid diffusion timescales of the order of seconds to minutes (at most), emphasising the syn-eruptive character of the mixing occurring throughout the conduit, possibly via the incorporation of ascending mafic magma pockets into a conduit filled by more silicic magma. This rare set of data testifies to the dynamic processes occurring in open magmatic systems.
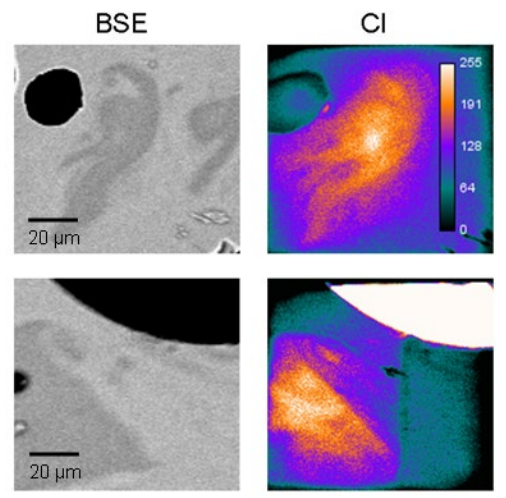

F
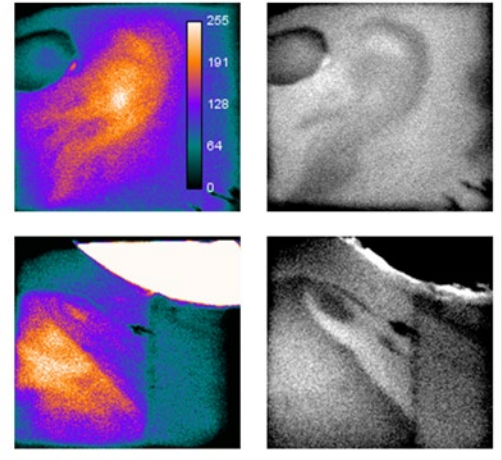